\title{
Effect of Hazelnut Oil on Muscle Cell Signalling and Differentiation
}

\author{
Ileana Terruzzi ${ }^{1}$, Fernanda Vacante ${ }^{2}$, Pamela Senesi ${ }^{2}$, Anna Montesano ${ }^{3}$, \\ Roberto Codella ${ }^{2,3}$ and Livio Luzi ${ }^{2,3 *}$ \\ ${ }^{1}$ Diabetes Research Institute, Metabolism, Nutrigenomics and Cellular Differentiation Unit, San Raffaele Scientific Institute, Milano, ITALY \\ ${ }^{2}$ Metabolism Research Center, IRCCS Policlinico San Donato, San Donato Milanese, Milano, ITALY \\ ${ }^{3}$ Department of Biomedical Sciences for Health, Università degli Studi di Milano, Milano, ITALY
}

\begin{abstract}
Nuts-enriched diets were shown to bear beneficial effects for human's health. Among nuts, hazelnut plays a major role in human nutrition and health because of its unique fatty acid composition (predominantly MUFA), fat soluble bioactives (tocopherols and phytosterols), vitamins (vitamin E), essential minerals (selenium), essential amino acids, antioxidant phenolics (caffeic acid), dietary fiber (soluble form), and bioactive phtytochemicals. The current study was designed to explore the cellular effects of two particular hazelnut strains (Ordu and Tonda).

Four hazelnut oils were obtained from 2 common strains (Ordu hazelnut oil, Ordu cuticle oil, Tonda "gentile" hazelnut oil, Tonda "gentile" cuticle oil). The metabolic and nutritional effects of the four hazelnut oils were assessed using an in vitro model of mouse myoblasts, identifying the intracellular mechanisms involved in muscle differentiation and in the modulation of specific muscle genes.

We demonstrated that hazelnut oils induced morphological changes in neo-formed myotubes increasing myotubes size. In particular, the diversified effects of the hazelnuts and cuticle oils on muscle fibres shape (on length and diameter respectively) determine a diversified pattern of action on elongation or hypertrophy of the muscle fibres. Furthermore, hazelnut oils regulate different pathways associated with myoblasts growth and development, stimulate signal transduction, and activate cell commitment and differentiation. The present results provide evidence that hazelnut oils may affect skeletal muscle growth and differentiation, constituting the proof of principle for the future development of novel foods and integrators.
\end{abstract}

Key words: hazelnut oils, skeletal muscle differentiation, novel foods, integrators

\section{Introduction}

Muscle is not only the major site of metabolic activity but it is the largest protein reservoir, providing a source of amino acids to be used for energy production during periods of food deprivation. Several studies have suggested that the loss of skeletal muscle may have serious health consequences. In contrast, physical activity, particularly resistance exercise, can generate large increases in skeletal muscle mass ${ }^{1)}$.

During the skeletal muscle differentiation, mononucleated myoblasts withdraw from the cell cycle and fuse into multinucleated myotubes ${ }^{2,3)}$, the basic cellular structures of muscle. Myogenesis is regulated by Myogenic Transcription Factors: MyoD, Myf5, Myogenin, Muscle Regulatory Factor 6(Myf6), and Myocyte specific Enhancer Factor 2 (MEF2) regulating the expression of muscle-specific genes, such as myosin heavy chain (MyHC) and creatine kinase ${ }^{4,5)}$.

Numerous reports previously addressed the intracellular pathways, downstream of hormonal(insulin) and nutritional signals, involved in skeletal muscle protein synthesis during differentiation and hypertrophy ${ }^{6,7)}$ revealing two major pathways by which these signals are transmitted:(1) the IRS1/phoshatidylinositol 3 kinase(PI3K)/AKT/p70 S6K pathway $^{8}$ and (2) the Ras/Raf/mitogen-activated protein kinase (MAPK) pathway ${ }^{9}$. PI3-Kinase activation is involved in cellular anti-apoptotic processes via AKT activation ${ }^{8}$, which is sufficient to induce hypertrophy in vivo and regulates the number, size and survival of mature muscle cells ${ }^{10,11}$. Downstream, the p70 S6 kinase phosphorylation starts translation process ${ }^{12)}$. A strong positive relationship between activation of p70 S6 kinase and the long-term increase in muscle mass has been observed in rat skeletal

*Correspondence to: Livio Luzi, Director, Endocrinology and Metabolism Policlinico San Donato, IRCCS, Via Morandi 30,20097 San Donato Milanese, Milano, ITALY

E-mail: livio.luzi@unimi.it

Accepted June 13, 2018 (received for review May 10, 2018)

Journal of Oleo Science ISSN 1345-8957 print / ISSN 1347-3352 online

http://www.jstage.jst.go.jp/browse/jos/ http://mc.manusriptcentral.com/jjocs 
muscle following resistance training ${ }^{1)}$. In the second pathway $^{9,13)}$, the activation of ERKs is necessary for the expression of muscle specific genes and phospho-ERK2 is essential for the formation of mature muscle fibres ${ }^{14)}$.

Skeletal muscle is intrinsically a stimulus-responsive tissue not only sensitive to the insulin stimulation, but also to the mechanical stimulus. Increased mechanical stretching is a powerful spur toward protein synthesis in the skeletal muscle. The PI3K/AKT/p70 S6 kinase and ERKs pathways, the same pathways stimulated by insulin, have been indicated to be of importance to the control of skeletal muscle mass, especially under mechanical stimulation ${ }^{9,10)}$.

Free fatty acids are the muscle preferred energy fuel in endurance exercises ${ }^{15)}$. Their accumulation into myofibrils was described in marathon runners ${ }^{16)}$ and endurance athletes $^{14)}$. Therefore, there is increasing evidence to suggest that fatty acids play a major role in regulating metabolism through effects on gene expression ${ }^{17)}$. Previous works have demonstrated that fatty acids may play an important role in regulating skeletal muscle growth ${ }^{17,18)}$.

The most studied source of unsaturated fatty acids is olive oil with its $80 \%$ content in oleic acid ${ }^{18)}$. Hazelnut oil has also a similarly high content of oleic acid, but it also contains several anti-oxidants and additional saturated and unsaturated fatty acids ${ }^{19,20)}$. Hazelnut oil is used much less in Mediterranean diet prescriptions, although its use is increasing in recent years.

The current study was designed to explore the cellular effects of four particular hazelnut oils (Ordu hazelnut oil, Ordu cuticle oil, Tonda gentile hazelnut oil, Tonda gentile cuticle oil-Table 1) using an in vitro model of mouse myoblasts, assessing the intracellular mechanisms involved in muscle differentiation and the modulation of muscle specific genes. Hazelnut plays a major role in human nutrition and health because of its unique fatty acid composition (predominating MUFA), fat soluble bioactives (tocopherols and phytosterols), vitamins (vitamin E), essential minerals (selenium), essential amino acids, antioxidant phenolic (caffeic acid), dietary fibre (soluble), and phytochemicals $^{20)}$.

\section{Experimental}

\subsection{Materials}

Anti-GAPDH(FL-335), anti p-ERK(E-4), anti p-p70 S6 kinase (Thr 421/Ser 424)-R, anti-MyoD (C-20), anti-Myf5 (C-20), anti-Myf6 (C-19), anti-Myogenin (D-10) and antiMyHC (H-300), monoclonal or polyclonal primary antibodies, the peroxidase-conjugated secondary antibodies and rhodamine-conjugated secondary antibodies were purchased from Santa Cruz Biotechnology (Santa Cruz, CA, U.S.A.). All other reagents were purchased from Sigma Chem. Co. (St. Louis, MO, U.S.A.) . Mouse C2C12 myoblasts cells were purchased from the European Collection of Animal Cell Cultures (ECACC).

\subsection{Cuticle oil extraction}

The hazelnut skin oil extractions were performed with a NATEX extractor (Natex, Extraction Co2 Pilot Unit 5.0 LTerniz, Austria). With NATEX extractor was possible to work in dynamic phase where the flux of $\mathrm{CO}_{2}$ was in continuous. FOOD 2 Carbon dioxide was used as solvent. A sample of about $1300 \mathrm{~g}$ has been loaded into the extraction reactor.

The start of extraction was set to achieve temperature and pressure of work and after a period of stabilization parameters. In the dynamic phase of supercritical $\mathrm{CO}_{2}$ extraction was observed the following conditions:

- Extractor pressure 300 bar

- Temperature $50^{\circ} \mathrm{C}$

- Flow $\mathrm{CO}_{2} \mathrm{Kg} / \mathrm{h} 30$, Tot solvent $120 \mathrm{~kg}$

After that, a cycle with reduced extraction time, pres-

Table 1 Schematic chemical composition of different hazelnut oils and olive oil analyzed in this study.

\begin{tabular}{|cccccc|}
\hline & OLIVE & OHO & OCO & TGHO & TGCO \\
\hline $\begin{array}{c}\text { Total Tocopherols } \\
\text { (mg/kg) }\end{array}$ & 402,32 & 690,99 & 1251,31 & 701,69 & 2320,70 \\
a tocopherol & 367,88 & 634,00 & 927,88 & 645,00 & 1092,00 \\
$\beta$ tocopherol & - & 14,02 & 125,00 & 14,49 & 117,26 \\
Y tocopherol & 20,94 & 35,88 & 0,00 & 35,65 & 1010,00 \\
$\delta$ tocopherol & - & 3,12 & 182,41 & 2,80 & 63,27 \\
Tocotrienols & 13,50 & 3,97 & 16,02 & 3,75 & 38,17 \\
\hline Mono/Poly Unsatured & 83,48 & 90,09 & 89,25 & 90,16 & 90,33 \\
Fatty acid \% & & & & & 9,05 \\
Satured Fatty Acids \% & 15,50 & 9,72 & 7,80 & 9,71 & 9,05 \\
Oleic acid \% & 72,37 & 82,28 & 75,60 & 82,36 & 78,70 \\
Total Sterols $(\mathrm{mg} / \mathrm{kg})$ & 1139 & 1572 & 3040 & 1541 & 3187 \\
\hline
\end{tabular}


sure and temperature was conducted.

Extraction yield was about $27 \%$ for Turkey hazelnut and $26 \%$ for Piedmont hazelnut.

\subsection{Kernel Oil extraction}

Hazelnut kernel oil extractions were performed with IR heating BROVIND pilot plant. Sample were first roasted, then ground in a disc mill. $350 \mathrm{~g}$ aliquots have been pressed using hydraulic press. Leaving oil temperature was about $41^{\circ} \mathrm{C}$.

Yields have been calculated by difference:

$[(\mathrm{Pc}-\mathrm{Ps}) /(\mathrm{Pc})] * 100$

$\mathrm{Pc}=$ initial weight sample (loaded into the press)

Ps $=$ dry sample weight (drained from the press)

Extraction yield was about $63 \%$ for Turkey hazelnut (Ordu hazelnut oil and Ordu cuticle oil) and 67\% for Piedmont hazelnut (Tonda gentile hazelnut oil and Tonda gentile cuticle oil). The main properties of oils used in this work are reported in Table 1.

\subsection{Experimental protocol}

C2C12 myoblasts were cultured at $37^{\circ} \mathrm{C}$ in humidified $5 \% \mathrm{CO}_{2}$ atmosphere in a growth medium (GM) containing DMEM supplemented with $20 \%$ (v/v) FBS (fetal bovine serum), 1\% penicillin-streptomycin and 1\% L-glutamine. Cell differentiation was initiated by placing $70 \%$ confluent cells cultures in DMEM supplemented with 1\% HS (horse serum) and antibiotics (DM). DM was changed every $24 \mathrm{~h}$. For the proliferating myoblasts experiments, the cells were grown to 40-50\% confluence in GM, for the myoblasts experiments the cells were cultured until to $70-80 \%$ confluence in GM, while for the myotube experiments C2C12 cells were differentiated in DM for $72 \mathrm{~h}$ (Fig. 1a). The cells used as control were maintained respectively in $\mathrm{GM}\left(\mathrm{C}_{\mathrm{GM}}\right)$ or in $\operatorname{DM}\left(\mathrm{C}_{\mathrm{DM}}\right)$. Other five groups of cells were placed in medium with the selective addition respectively of Ordu hazelnut oil (OHO; 10\%) or of Ordu cuticle oil (OCO; 10\%), of Tonda gentile hazelnut oil (TGHO; $10 \%$ ) or of Tonda gentile cuticle oil (TGCO; 10\%), of commercial olive oil (OLIVE; 10\%). Cells were lysed at 30, 60 and $240 \mathrm{~min}$ after the stimuli addition. For Immunofluorescence analysis, C2C12 differentiated myocytes were stimulated for $4 \mathrm{~h}$.

\subsection{RT-PCR array analysis}

Gene expression levels were assessed by RT2 -PCR Arrays System. In general, RT2 -PCR Array plates produced by SABiosciences (SABiosciences Corporation, Frederick, MD 21703 USA) were designed to analyze simultaneously a panel of genes. We decided to study the following gene expression: MyoD, Myf5, Myf6, Myogenin, Myostatin, MyHC, p21, Rb, cyclin D and cyclin B1. GAPDH/ Actin housekeeping genes were included as endogenous normalization controls. Total RNA was isolated from C2C12 cells using the RNeasy Plus Mini Quiagen kit(Quiagen
GmbH, Germany) according to the manufacturer's instructions. Total RNA $(1 \mu \mathrm{g})$ was reverse transcribed using RT2 First Strand Kit (SABiosciences Corporation, Frederick, MD 21703 USA). The reverse transcripts were used as templates for analysis of gene expression level using RT2-PCR Arrays plates according to the manufacturer's instructions. Each sample was run in triplicate. The expression level of the housekeeping genes chosen for normalization in the threshold cycle (Ct) for each experimental condition and then the fold-change $(\Delta \Delta \mathrm{Ct})$ for each gene from treatment group compared to the control group was calculated. If the $\Delta \Delta \mathrm{Ct}$ is greater than 1 , then the result may be reported as a fold up-regulation. If the $\Delta \Delta \mathrm{Ct}$ is less than 1 , then the result may be reported as a fold down-regulation.

\subsection{Immunoblotting analysis}

C2C12 extracts were prepared by lising the cells in RIPA buffer at $4{ }^{\circ} \mathrm{C}^{5)}$. Aliquots of $30 \mu \mathrm{g}$ supernatant proteins were separated with SDS-polyacrylamide gel electrophoreses (SDS-PAGE) and electrophoretically trasferred to nitrocellulose membranes (Protran, Whatman Schleicher \& Schuell) using a Bio-Rad Mini Trans-Blot system. Blots were blocked and then incubated with respective primary antibodies and then with species-specific secondary antibodies. To confirm equal protein loading per sample, we used antibody anti-GAPDH. Immunoreactive bands were visualized by an enhanced chemiluminescence method (Amersham Pharmacia Biotech, Piscataway, NJ, USA) and quantified by densitometric analysis using the Scion image software (Scion corporation, Frederick, MD, USA). Data were then converted into fold-changes $(\mathrm{FC})$ of the controls.

\subsection{Immunofluorescence analysis}

Proliferating myoblasts or differentiated myotubes were fixed in prepared $4 \%$ paraformaldehyde and, then, maintained in PBS. The resulted cells were permeabilized with $0.2 \%$ Triton X-100 and blocked with PBS containing 1\% bovine serum albumin(BSA). Then, cells were incubated with primary antibodies. To detected primary antibody binding sites, cells were washed in PBS and followed by incubation with specific antibodies rhodamine-conjugated. Coverslips with cells were mounted with Moviol and observed using Nikon Eclipse 50I fluorescence microscopy and images were captured using Nis-Elements D 4.00 software.

\subsection{Statistical analysis}

All experiments were performed three times. The data are presented as the mean \pm standard deviation and statistical comparisons were performed with specific statistical packages (Prism v 7.00 GraphPad Software, San Diego, CA, USA). Statistically significant differences were determined using ANOVA test followed by Tukey's multiple comparison 
A
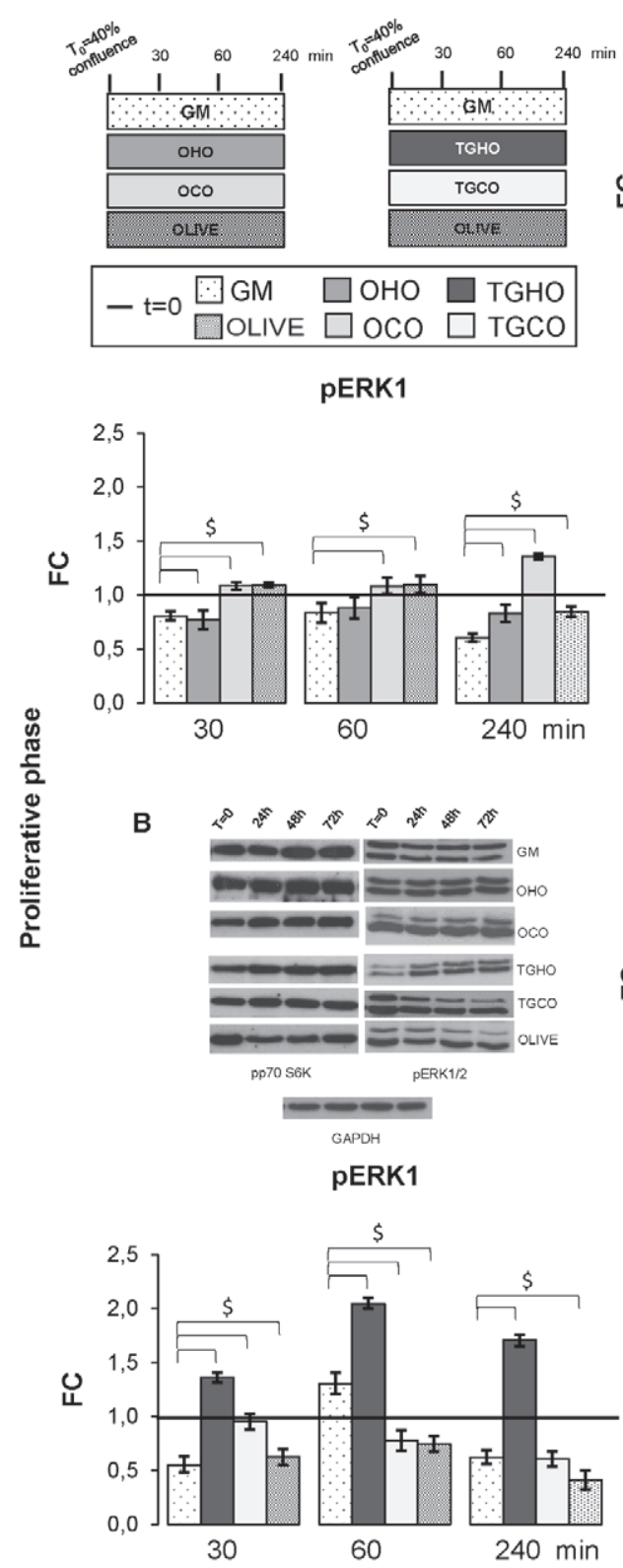

pp70 S6K
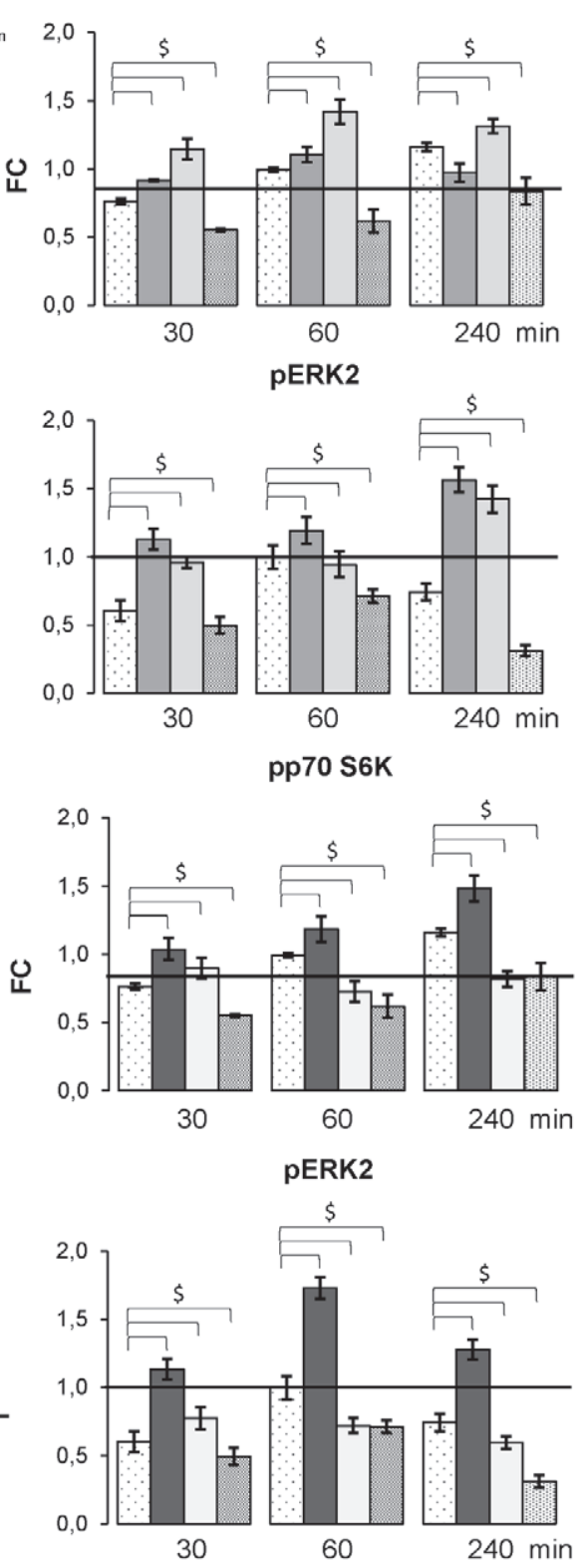

Fig. 1 During proliferative phase, OCO (A) or TGHO (B) promoted p70 S6 kinase activation, while OHO, OCO (A) or TGHO (B) increased ERK1/2 phosphorylation at $240 \mathrm{~min}$. Representative immunoblots are shown. Tukey post test: $\S p \leq 0.001$ vs $\mathrm{GM}_{40 \%}$ confluence.

test.

Results were considered statistically significant when $p$ $\leq 0.05$.

\section{Results}

In the experiments, we tested the ability of both hazelnut oils (OHO-OCO / TGHO-TGCO) and of a common commercial extra-virgin olive oil(OLIVE) to stimulate the same pathways in myoblast un-differentiated cells.

\subsection{Effects of hazelnut oils during proliferative phase}

In the proliferative phase, we investigated whether hazelnut and olive oils were able to induce protein synthesis by the activation of p70 S6 kinase ${ }^{1)}$. OCO (Fig. 1A) or TGHO

(Fig. 1B) treatments caused a significant p70 S6 kinase phosphorylation increase (30, 60 and $240 \mathrm{~min}$ ), while $\mathrm{OHO}$

(Fig. 1A), TGCO (Fig. 1B) or OLIVE oil did not modify p70 S6 activity. 


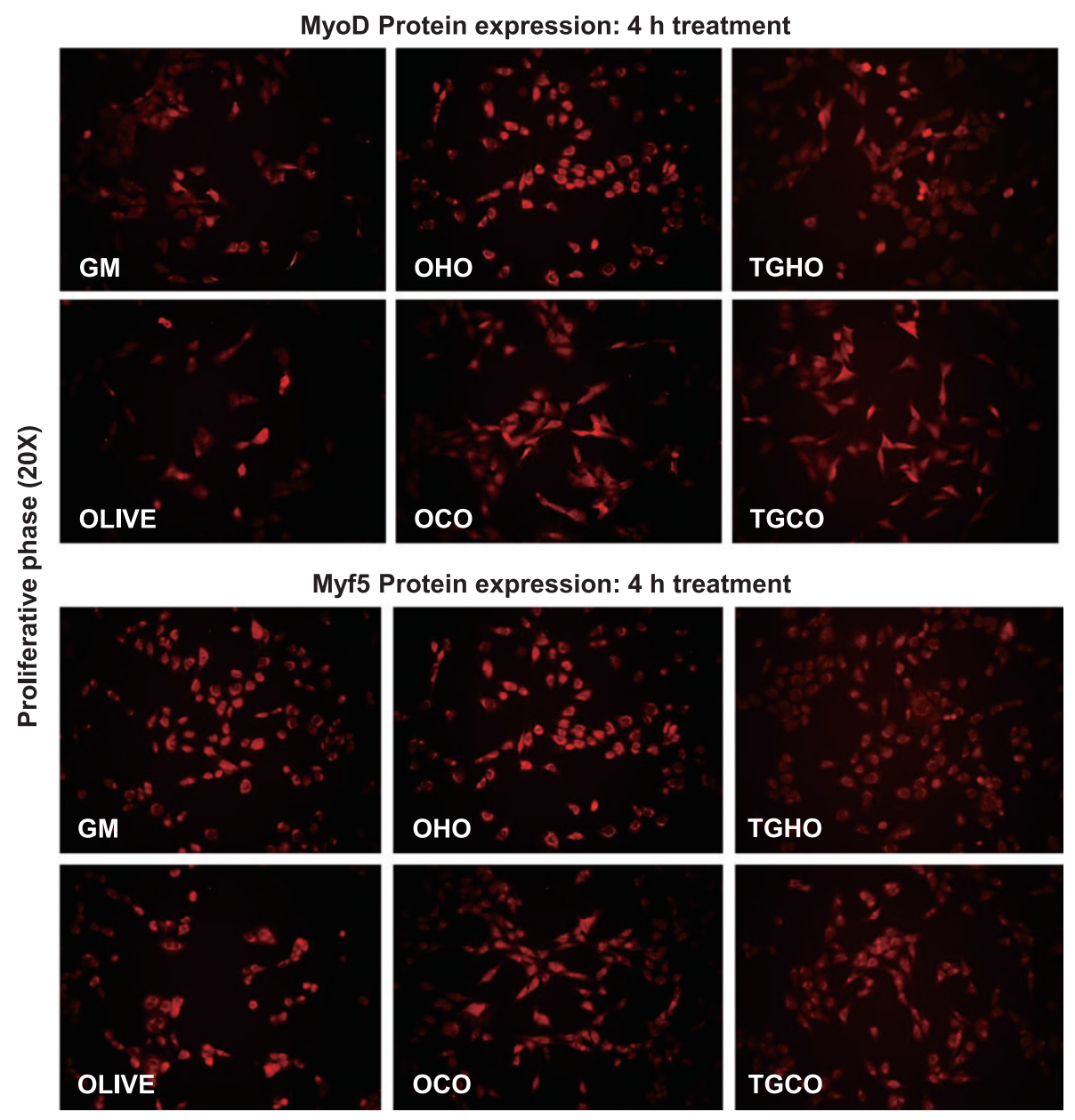

Fig. 2 C2C12 myoblast in proliferation phase were treated for $4 \mathrm{~h}$ with $10 \%$ different oils. The OHO or TGHO treatments promoted the MyoD and Myf5 expression in respect to the control cells (GM). The cuticle oils (OCO and TGCO)not only increased the levels of these proteins, but also induced the first morphologic changes to transforming myoblasts in myocyte cells.

Moreover, we analyzed the Ras/MEK/ERK cascade involved in mitosis and cell proliferation. We determined the levels of ERK1 and ERK2 phosphorylation ${ }^{7)}$ : at $240 \mathrm{~min}$, ERK1 and ERK2 were activated by OCO, OHO (Fig. 1A) and TGHO oils (Fig. 1B). These results suggest how in myoblast cells hazelnut oils could positively induce myocyte commitment in respect to the OLIVE.

To confirm this preliminary evidence, we investigated MyoD and Myf5 protein expression in C2C12 myoblast cells by Immunofluorescence analysis ${ }^{4}$ after $4 \mathrm{~h}$ of oils treatment: our data suggested that OCO and TGCO induced the first morphologic changes to commit myoblasts in myocyte cells (Fig. 2) .

\subsection{Effects of hazelnut oils during differentiation phase}

Since myocytes differentiation requires cell cycle withdrawal $^{2,3)}$, we studied the gene expression of cell cycle regulators and myogenic regulatory factors (MRFs) MyoD, Myf5, Myf6 and Myogenin in myoblast cells after 4(Fig.
3A) and $48 \mathrm{~h}$ (Fig. 3B) of oils treatment. As reported in Experimental section, if the $\Delta \Delta \mathrm{Ct}$ is greater than one, then the result can be reported as a fold up-regulation. If the $\Delta \Delta \mathrm{Ct}$ is less than one, then the result can be reported as a fold down-regulation.

We observed the increase of key cell cycle regulators and of MRFs gene expression after 4(Fig. 3A) and $48 \mathrm{~h}$ (Fig. 3B) of hazelnut oils supplementation. In contrast, the expression of these MRFs was not detected in cells treated with OLIVE.

These results indicate that treatments with hazelnuts oils promoted the gene expression of MRFs ${ }^{17}$ and skeletal muscle specific protein MyHC not only in the early phase (Fig. 3A) but also during differentiation (Fig. 3B). To confirm this observation and clarify the degree of myogenic differentiation induced by oils stimulation, we studied the effects on protein synthesis by Western Blot and Immunofluorescence analysis.

Subsequently, we examined the effect of oils during the 
Gene expression: $4 \mathrm{~h}$ treatment

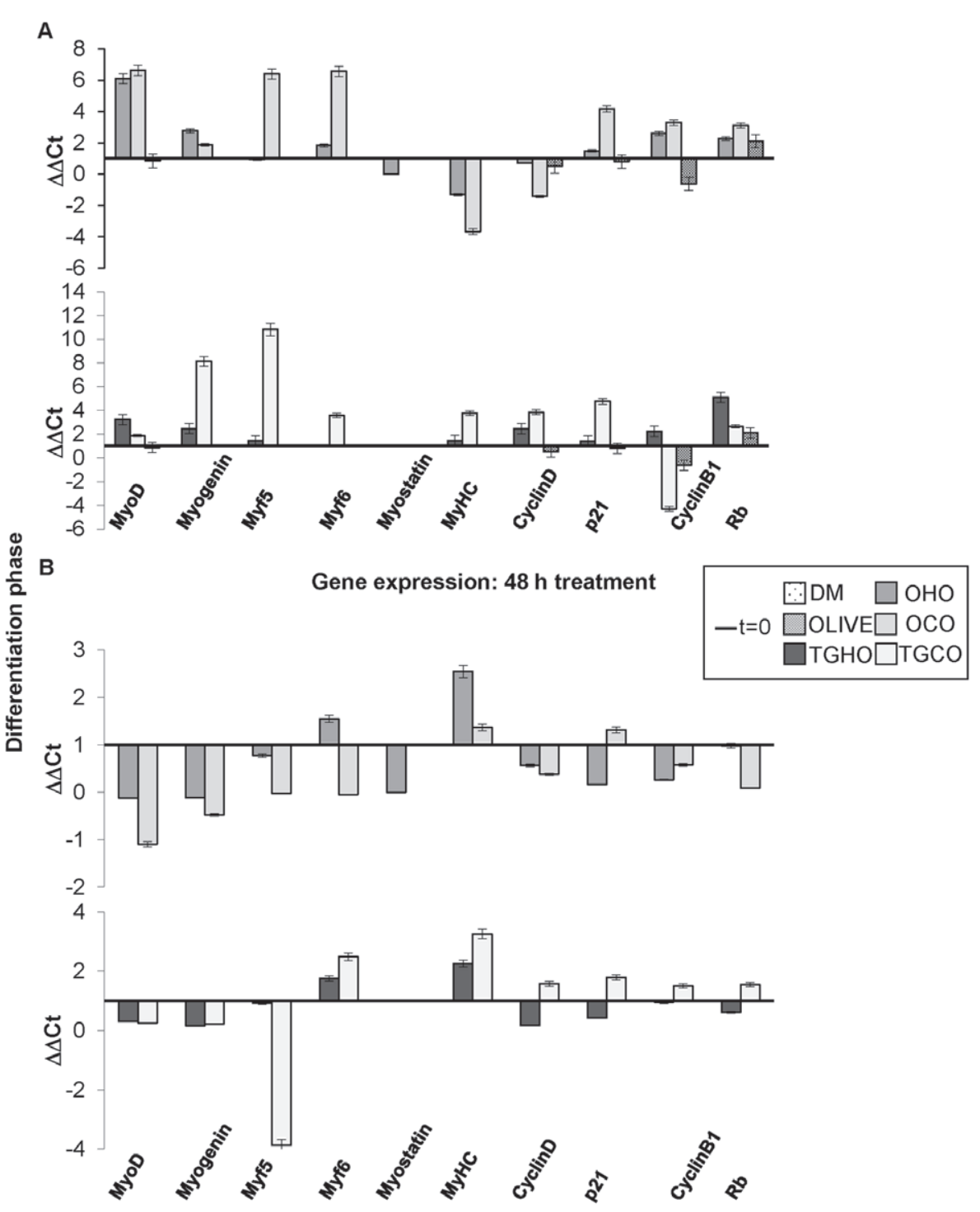

Fig. 3 During differentiation phase, hazelnut oils supplementation increased the key cell cycle regulators and MRFs gene expression after 4 and $48 \mathrm{~h}$ of oils treatments. In contrast, the expression of these MRFs was not detected in cells treated with OLIVE.

differentiation phase. After $24 \mathrm{~h}$ of differentiation in presence of OLIVE oils, most of cells showed a markedly reduced vitality at $48 \mathrm{~h}$, the total $\mathrm{C} 2 \mathrm{C} 12$ cells were died. We did not observe any toxic effects in presence of hazelnut oils.

First, we studied the activation of the principal protein kinases involved in protein synthesis and muscle differentiation ${ }^{12)}$. As shown in Fig. 4, all hazelnut oils induced p70 S6 activation (Figs. 4A, 4B).

ERK1/2 play an important role during muscle differentiation and hypertrophy ${ }^{10)}$. Hazelnut and cuticle supplements increased ERK1/2 activation at all time-points (Figs. 4A, 4B). These results indicated that hazelnut oils could modulate specific signaling pathways involved in myogenic factors synthesis.

In fact, OCO, TGHO or TGCO treatment increased MyoD protein level (Figs. 5A, 5B) and all hazelnut oils increased Myogenin protein levels in respect with untreated $\mathrm{C} 2 \mathrm{C} 12$ (DM). We determined MyHC synthesis: at the end of differentiation, MyHC levels were the same in the negative control (DM) and in treated cells with $\mathrm{OCO}, \mathrm{OHO}$ or TGCO (Fig. 5A), while TGHO increased MyHC protein levels (Fig. $5 B)$.

\subsection{Effects of hazelnut oils on post-differentiation hyper- trophy}

To clarify hazelnut oils action on hypertrophy process, neo-formed myotubes were treated for 30, 60 and $240 \mathrm{~min}$ 

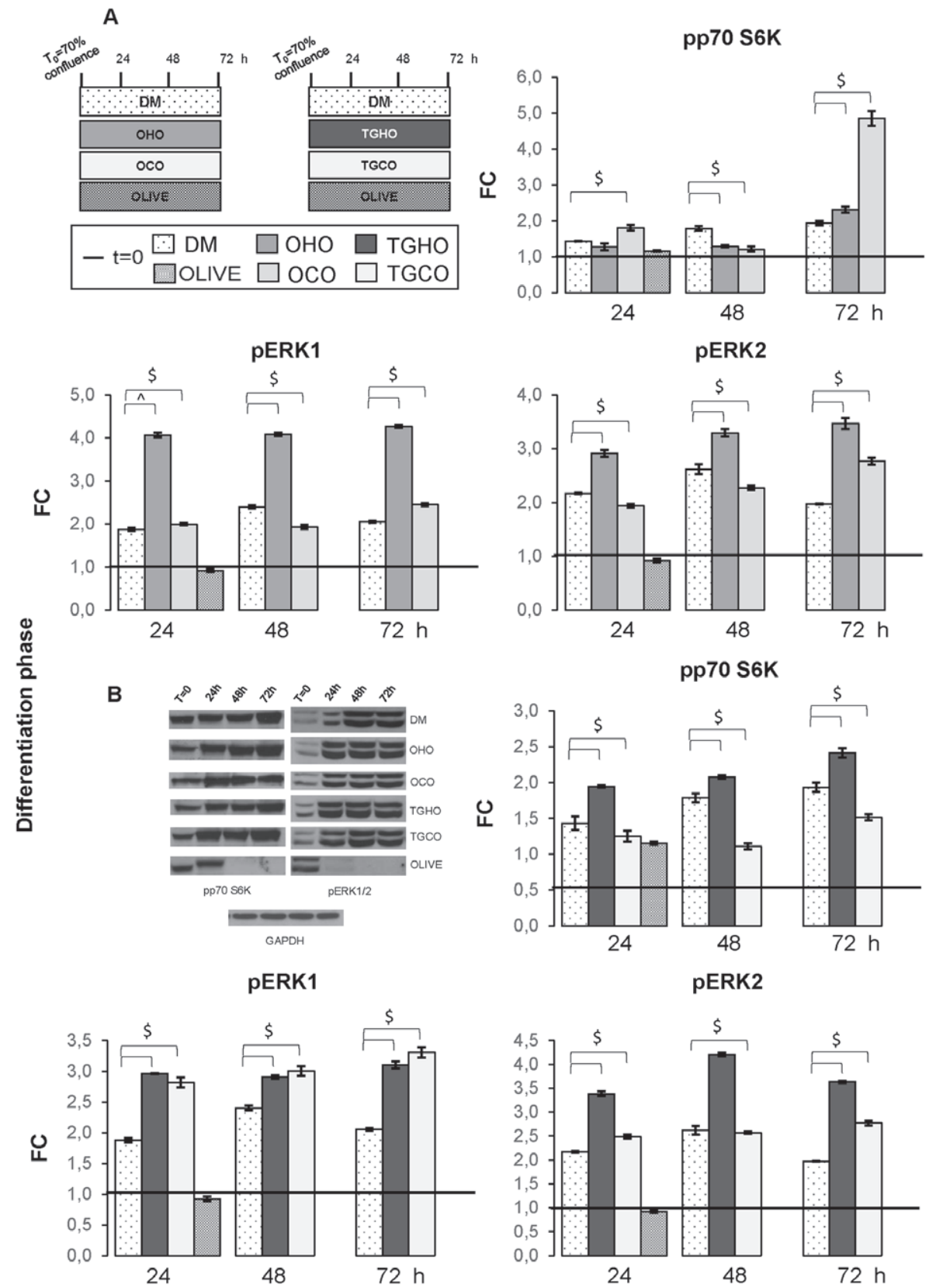

Fig. 4 After $48 \mathrm{~h}$ of differentiation in presence of OLIVE oil, the total C2C12 cells were died. All hazelnut oils increased p70 S6 kinase and ERK1/2 activation at all time point (A, B). Representative immunoblots are shown. Tukey post test: $\wedge p \leq 0.002, \S p \leq 0.001$ vs $\mathrm{DM}_{70 \%}$ confluence.

with the five oils after $72 \mathrm{~h}$ differentiation.

We observed a strong increase of p70 S6 kinase phosphorylation after $30 \mathrm{~min}$ OHO or TGCO stimuli(Figs. 6A, 6B). In contrast, OLIVE oil significantly decreased p70 S6 kinase phosphorylation level. Furthermore, we observed a weak modulation of ERKs pathway ${ }^{10)}$ : at $240 \mathrm{~min}$, OCO and TGCO oils promoted ERKs phosphorylation (Figs. 6A, 6B).

We examined neo-formed myotubes morphologic features. Images of MyHC-positive myotubes, detected by Immunofluorescence after $4 \mathrm{~h}$, showed that OLIVE oil nega- tively influenced myotubes formation. In contrast, cells stimulated with $\mathrm{OHO}$ showed an increase of the diameter respect DM, while neo-formed myotubes stimulated with OCO or TGCO were longer compared to DM(Fig. 7A).

This evidence was further confirmed by Myf6 Immunofluorescence assay (Fig. 7B): OCO, TGHO and TGCO oils showed a major number of Myf6 positive myotubes.

Taken together, these results indicate that hazelnuts oils can induce the activation of signaling pathways involved in muscle differentiation and hypertrophy and, in contrast to 
A
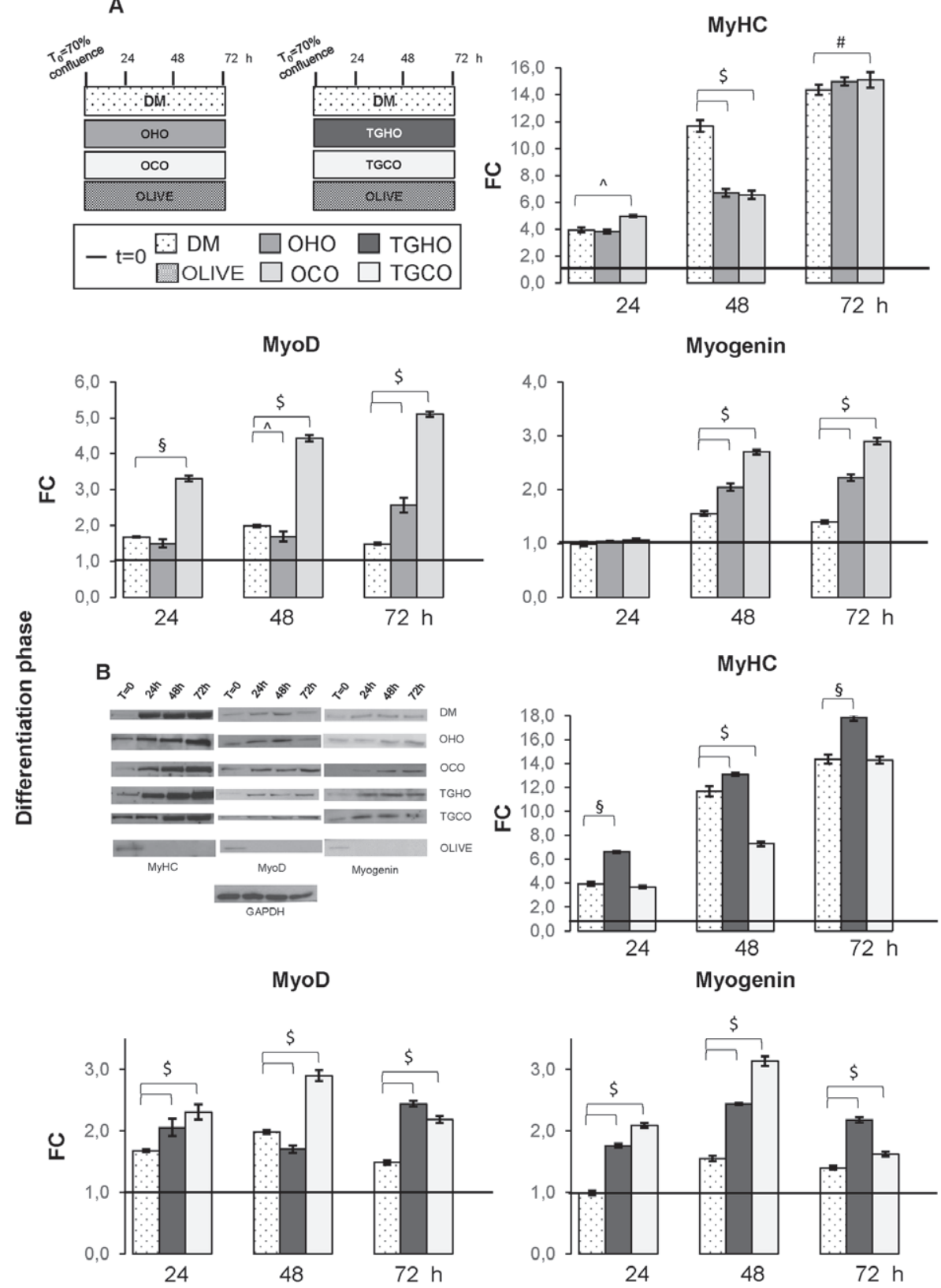

Fig. 5 During differentiation phase, in OCO or TGCO cells MyoD levels were significantly increased in respect of DM(A, B). Hazelnut oils supplementation increased Myogenin protein levels in relation to DM(A, B). MyHC levels at the end of differentiation were the same in the control DM and in treated cells with OCO, OHO or TGCO while TGHO increased MyHC protein levels. Representative immunoblots are shown. Representative immunoblots are shown. Tukey post test: $\# p \leq 0.04, \wedge p \leq 0.002, \S p \leq 0.001 \mathrm{vs} \mathrm{DM}_{70 \% \text { confluence. }}$.

common commercial OLIVE, can promote the different phases of muscle differentiation process (Table 2).

\section{Discussion}

The skeletal muscle mass is closely related to the number of cells which trigger the myogenic program and are able to undergo hypertrophy at later stages of muscle development ${ }^{3,9)}$. The nutritional and humoral factors controlling skeletal muscle mass and function acting during development deserve particular attention. In fact, muscle hypotrophy/atrophy (sarcopenia) is an important clinical problem, negatively impacting human health. Chronic inactivity (such as prolonged bed rest), accelerates the aging process $^{21)}$. Poor nutrition and physical inactivity are the $2^{\text {nd }}$ 
A
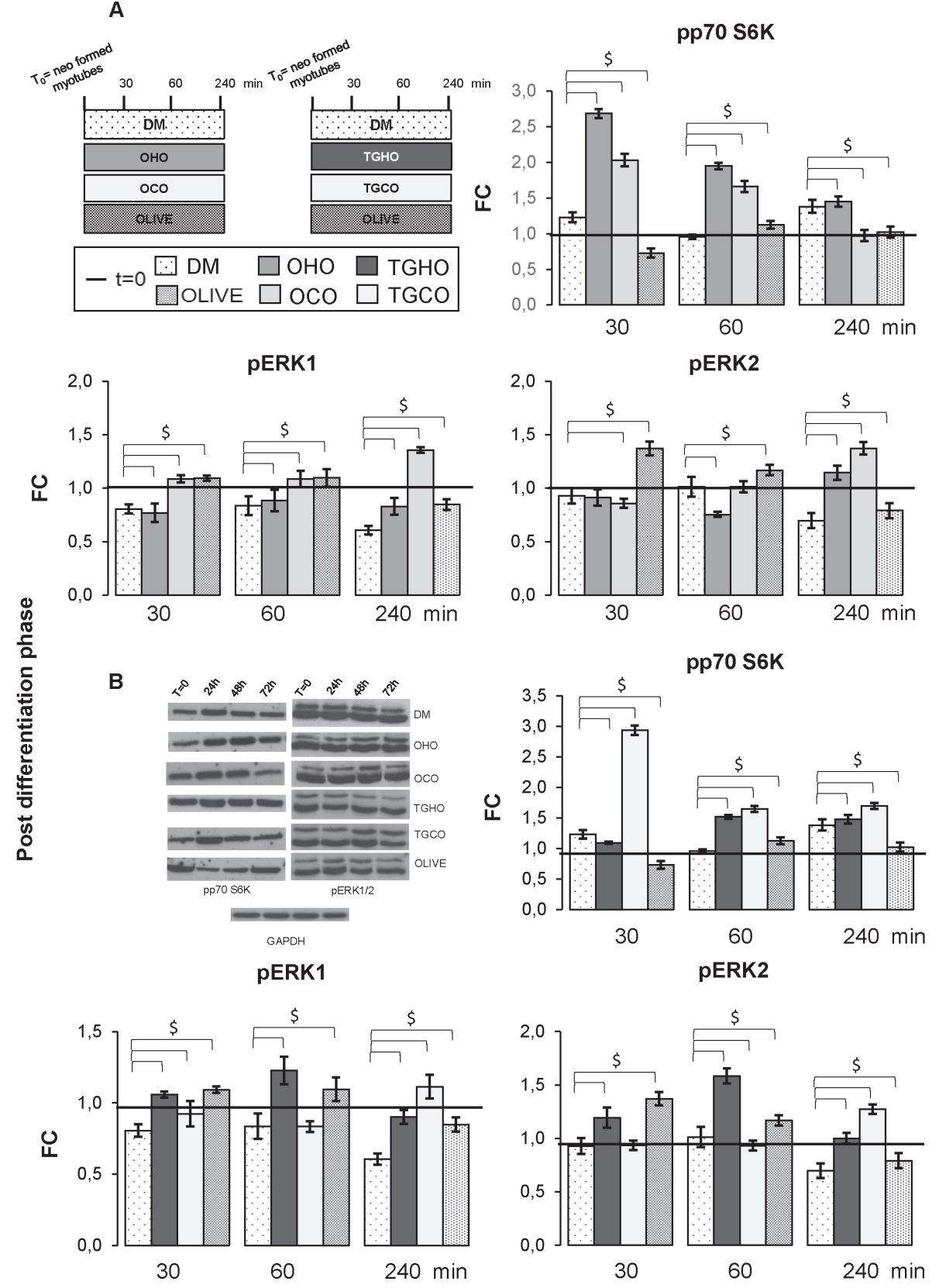

Fig. 6 In neo-formed myotubes, OHO or TGCO induced a strong increase of p70 S6 kinase phosphorylation after 30 min of stimulation. OLIVE oil significantly decreased p70 S6 phosphorylation level(A, B). At 240 min, OCO and TGCO oils promoted ERKs phosphorylation (A, B). Representative immunoblots are shown. Tukey post test: $\S p \leq 0.001$ vs $\mathrm{DM}_{\text {post-diff. }}$

cause of mortality (next to cigarette smoking) in humans (Center of Diseases Control, Atlanta, GA). Currently, dietary recommendations and physical exercise counselling are the prevailing means to counteract muscle hypotrophy/ atrophy.

Among dietary nutrients, amino acids were known since decades to stimulate protein anabolism and to mimic insulin effect activating intra-cellular signals into several cell types $^{22)}$. Overall, the effect of amino acids is compara- ble to the one of high protein diet, although branchedchain amino acids seem to have the highest insulin-mimetic and trophic activity in skeletal cells ${ }^{23,24)}$. Similar effects of high-fat diets and single fatty acids integration on muscle accretion are much less known. Chanseaume and others ${ }^{25)}$ demonstrated that an increase muscle mixed and mitochondrial protein synthesis rates after a high-fat or highsucrose diet. Very recently, Rincón-Cervera and others ${ }^{26)}$ showed that supplementation with extra-virgin olive oil 
A

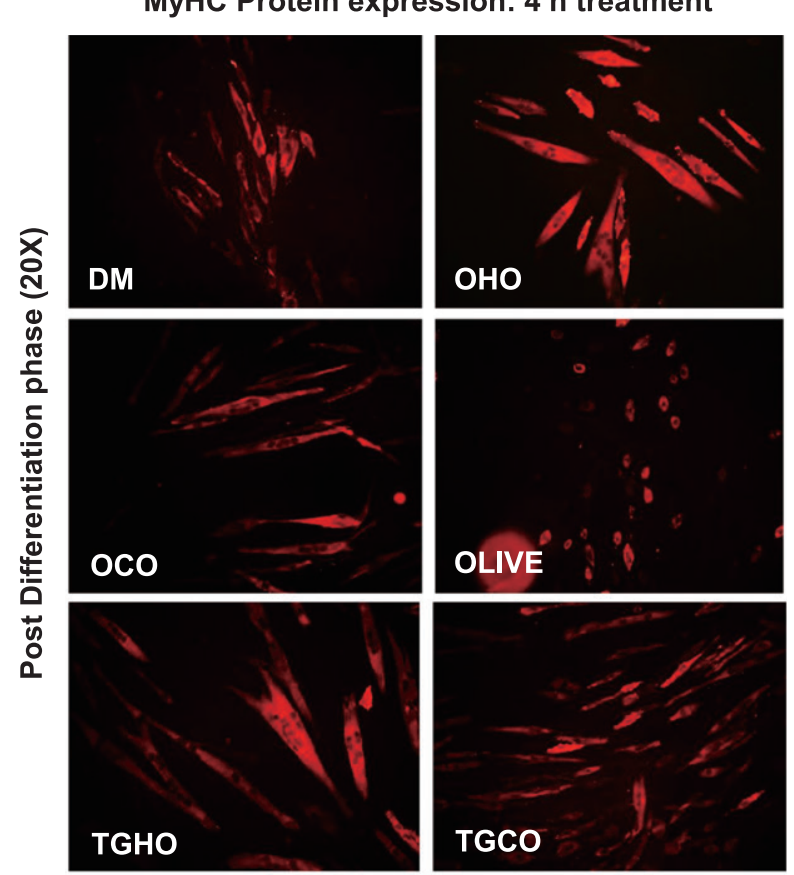

Myf6 Protein expression: $4 \mathrm{~h}$ treatment

B

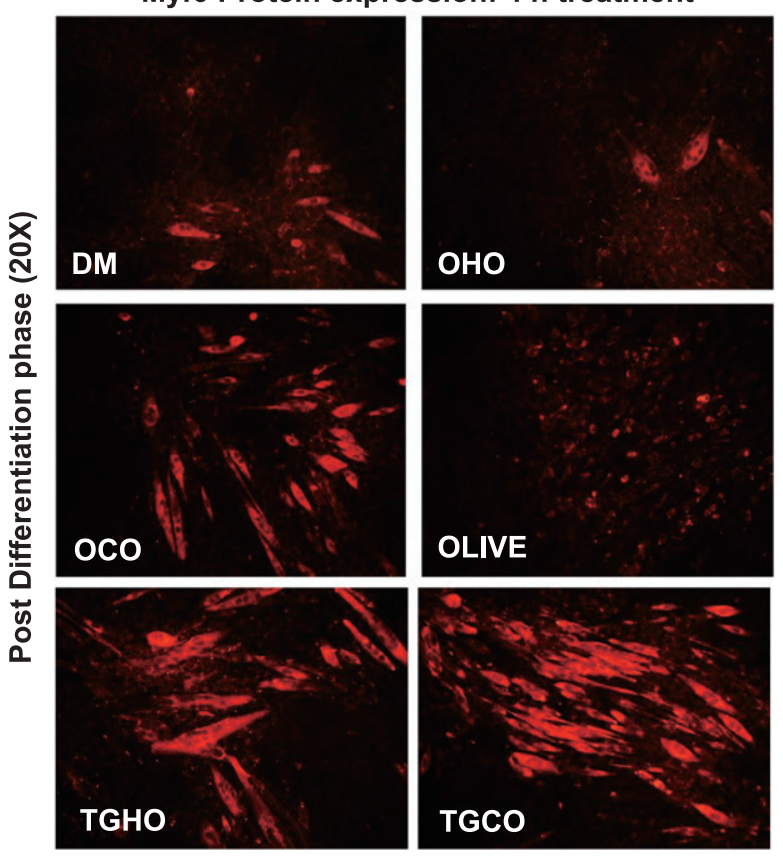

Fig. 7 In neo-formed myotubes, OHO cells showed an increase of the diameter with respect to the control DM. The cells stimulated with OCO or TGCO showed a rise in the length in MyHC positive myotubes compared with the un-stimulated differentiated cells (DM) (A). OCO, TGHO and TGCO treatments showed a major number of Myf6 positive myotubes $(\mathrm{B})$. prevents hepatic oxidative stress and reduction of desaturation capacity in mice fed a high-fat diet.

We presently report the effects of hazelnut oils on intracellular pathways and myotubes differentiation in a model of murine myoblasts. This is the first study to describe the effects of hazelnut oil on Myogenic Regulatory Factors (MRFs) expression during differentiation in C2C12 skeletal muscle cells. We presently show that hazelnut oil integration is able to stimulate myogenic phenotype acquisition and differentiation process through MRFs protein expression in $\mathrm{C} 2 \mathrm{C} 12$ in vitro model.

Concerning the mechanism (s), our data suggest that the activation of ERKs pathways and the strong activation of kinases involved in the protein synthetic pathway(AKT and $\mathrm{p} 70)^{6,10)}$ appear to be responsible of an enhanced differentiation process with a selective stimulation in the proliferative (Fig. 1) differentiation (Fig. 4) and post-differentiation(Fig. 6) phases. This is a novel mechanism, not common to other "healthy" oils as olive oil. In fact, olive oil, used as control, showed a scarce or absent effect on proliferation, differentiation and post-differentiation phase of myoblasts. A previous study described the reduction of fat mass with an increase of muscle mass following the assumption of 12 table green olives/day for 30 days $^{27)}$. We presently use commercial standard olive oil as control, whilst Accardi et al used extra-virgin olive oil ${ }^{27)}$. Moreover, the experimental set-up (whole-body vs. murine cell models) used in the 2 studies was significantly different. However, the differential effect on muscle cell metabolism/ homeostasis between hazelnut oil and olive oil is noteworthy and may be explained as follows: 1) hazelnut oil has a higher content of oleic acid with respect to standard olive oil; 2) the polyphenols content and total anti-oxidant power is definitely higher in hazelnut oil with respect to standard olive oil. Since the PREDIMED trial showed that dietary polyphenols trigger nitric oxide (NO) production ${ }^{28)}$, it is conceivable that a more pronounced NO release, besides having the well-known vasodilatory effect, might also contribute to cell proliferation as previously demonstrated in cardiomyoblasts $^{29)}$.

Del Rio and others ${ }^{30)}$ previously reported on the 3-fold higher anti-oxidant power of cuticle with respect to the body in hazelnuts. Therefore, we tested also oils derived only by cuticle of hazelnut on cellular signals as well as cell differentiation and proliferative activity. All four hazelnut oils induced morphological changes in neo-formed myotubes increasing myotubes size. Nonetheless, OCO and TGHO have the stronger effect in activating p70 S6 kinase and pERK2 at 60 and 240 min of the proliferative phase. This indicates the 2 hazelnut oils as potential candidates to be used as novel foods or to develop and test food integrators with the specific target of stimulating protein anabolism. For instance, poorly-controlled diabetes mellitus is characterized by increased protein catabolism ${ }^{31)}$, reduced 
Table 2 Table resuming the effects of the 5 oils tested in this study during: a) Proliferative stage, b) Differentiation phase, and c) Post-differentiation hypertrophy. $\boldsymbol{\Delta}$, increase; $\boldsymbol{\nabla}$, decrease; =, no effects vs Control.

\begin{tabular}{|c|c|c|c|c|c|c|c|c|c|c|c|c|c|}
\hline & \multicolumn{3}{|c|}{ Proliferative stage } & \multicolumn{5}{|c|}{ Differentiation phase } & \multicolumn{5}{|c|}{$\begin{array}{c}\text { Post-differentiation } \\
\text { hypertrophy }\end{array}$} \\
\hline & \multirow[t]{2}{*}{ pp70 } & \multirow[t]{2}{*}{ pERK } & \multirow[t]{2}{*}{ Myocite Commitment } & \multirow[t]{2}{*}{ pp70 } & \multirow[t]{2}{*}{ PERK } & \multicolumn{3}{|c|}{ Myogenic factors synthesis } & \multirow[t]{2}{*}{$\mathrm{pp} 70$} & \multirow[t]{2}{*}{ PERK } & \multicolumn{3}{|c|}{ Myogenic factors synthesis } \\
\hline & & & & & & Myod & Myogenin & Мунс & & & Diameter & Length & Myt6 \\
\hline OLIVE & $\mathbf{v}$ & $\mathbf{v}$ & $=$ & & & Cell $\mathrm{c}$ & & & $=$ & $=$ & $=$ & $=$ & $=$ \\
\hline $\mathrm{OHO}$ & $=$ & $\Delta$ & $=$ & $\Delta$ & $\Delta$ & $=$ & $\Delta$ & $=$ & $\Delta$ & $=$ & $\Delta$ & $=$ & $=$ \\
\hline Oco & $\Delta$ & $\Delta$ & $\Delta$ & $\Delta$ & $\Delta$ & $\Delta$ & $\Delta$ & $=$ & $=$ & $\Delta$ & $=$ & $\Delta$ & $\Delta$ \\
\hline TGHO & $\Delta$ & $\Delta$ & $=$ & $\Delta$ & $\Delta$ & $\Delta$ & $\Delta$ & $\Delta$ & $=$ & $\Delta$ & $=$ & $=$ & $\Delta$ \\
\hline TGCO & $\nabla$ & $\nabla$ & $\Delta$ & $\nabla$ & $\Delta$ & $\Delta$ & $\Delta$ & $=$ & $\Delta$ & $\Delta$ & $=$ & $\Delta$ & $\Delta$ \\
\hline
\end{tabular}

muscle mass and muscle fibers strength. The present in vitro data constitute a proof of principle for testing specific hazelnut oils strains and extraction methodologies (mainly $\mathrm{OCO}$ and $\mathrm{TGHO}$ ) as potential food integrators to counteract protein catabolism in diabetic patients.

An important issue to be considered is whether the effect of OCO and TGHO may be mediated by epigenetic modifications induced by a hazelnut oil enriched diet over the decades. In a previous study, Surra and others ${ }^{32}$ demonstrated that, dietary nut supplementation delays the development of atherosclerotic lesions in female apoE-deficient mice, increasing the mRNA expression of genes involved in non-HDL cholesterol synthesis. Additional studies are required to specifically address this issue in our experimental setting.

\section{Conclusion}

The present study provide an in vitro evidence in a model of murine myoblast of a strong effect of hazelnut oils derived by the body and the cuticle of two common strains of hazelnuts (Ordu and Tonda) on myoblasts differentiation and cell muscle hypertrophy. In recent years, several studies have suggested that the damage of skeletal muscle may have serious health consequences. Currently, dietary recommendations and physical exercise counselling are the prevailing means to counteract muscle hypotrophy/ atrophy. In particular, our data suggest a potential use of hazelnut oils (or components of them) as food integrators to prevent muscle catabolism and atrophy.

\section{Acknowledgements}

The research was carried out thanks to the financial support of Soremartec Italia Srl(Alba, Cuneo, Italy). The funder had no role in study design, data collection and analysis, decision to publish or preparation of the manuscript.

\section{References}

1) Baar, K.; Esser, K. Phosphorylation of $p 70^{\mathrm{S} 6 \mathrm{k}}$ correlates with increased skeletal muscle mass following resistance exercise. Am. J. Physiol. 276, c120-c127 (1999).

2) De Falco, M.; De Luca, A. Involvement of cdks and cyclins in muscle differentiation. Eur. J. Histochem. 50, 19-23 (2006).

3) Sabourin, L.A.; Rudnicki, M.A. The molecular regulation of myogenesis. Clin. Genet. 57, 16-25 (2000).

4) Dedieu, S.; Mazères, G.; Cottin, P. et al. Involvement of myogenic regulator factors during fusion in the cell line C2C12. Int. J. Dev. Biol. 46, 235-241 (2002).

5) Montesano, A.; Luzi, L.; Senesi, P. et al. Resveratrol promotes myogenesis and hypertrophy in murine. $J$. Transl. Med. 11, 310(2002).

6) Rommel, C.; Bodine, S.C.; Clarke, B.A. et al. Mediation of IGF-1-induced skeletal myotube hypertrophy by PI (3) K/Akt/mTOR and PI(3) K/Akt/GSK3 pathways. Nat. Cell Biol. 3, 1009-1013 (2001).

7) Lee, J.; Hong, F.; Kwon, S. et al. Activation of p38 MAPK induces cell cycle arrest via inhibition of Raf/ ERK pathway during muscle differentiation. Biochem. Biophys. Res. Commun. 298, 765-771(2002).

8) Wilson, E.M.; Rotwein, P. Selective control of muscle differentiation by Akt. J. Biol. Chem. 282, 5106-5110 (2007).

9) Sandri, M. Signaling in muscle atrophy and hypertrophy. Physiology (Bethesda) 23, 160-170 (2008).

10) Li, J.; Johnson, S.E. ERK2 is required for efficient terminal differentiation of skeletal myoblasts. Biochem. Biophys. Res. Commun. 345, 1425-1433 (2006).

11) Lluis, F.; Perdiguero, E.; Nebreda, A.R. et al. Regulation of skeletal muscle gene expression by p38 MAP kinases. Trends Cell Biol. 16, 36-44(2006).

12) Long, Y.C.; Widegren, U.; Zierath, J.R. Exercise-induced mitogen-activated protein kinase signalling in skeletal muscle. Proc. Nutr. Soc. 63, 227-232 (2004).

13) Jeppesen, J.; Kiens, B. Regulation and limitations to fatty acid oxidation during exercise. J. Physiol. 590, 


\section{Terruzzi, F. Vacante, P. Senesi et al.}

1059-1068(2012).

14) Gondret, F.; Combes, S.; Lefaucheur, L. et al. Effects of exercise during growth and alternative rearing systems on muscle fibers and collagen properties. Reprod. Nutr. Dev. 45, 69-86 (2005).

15) Lee, J.H.; Tachibana, H.; Morinaga, Y. et al. Modulation of proliferation and differentiation of $\mathrm{C} 2 \mathrm{C} 12$ skeletal muscle cells by fatty acids. Life Sci. 84, 415-420 (2009).

16) Rapoport, B.I. Metabolic factors limiting performance in marathon runners. PLoS Comput. Biol. 6, e1000960 (2010).

17) Hurley, M.S.; Flux, C.; Salter, A.M. et al. Effects of fatty acids on skeletal muscle cell differentiation in vitro. Br. J. Nutr. 95, 623-630 (2006).

18) Degirolamo, C.; Rudel, L.L. Dietary monounsaturated fatty acids appear not to provide cardioprotection. Curr. Atheroscler. Rep. 12, 391-396 (2010).

19) Li, H.; Parry, J.W. Phytochemical compositions, antioxidant properties, and colon cancer antiproliferation effects of Turkish and Oregon hazelnut. Food Nutr. Sci. 2, 1142-1149 (2011).

20) Cesarettin, A.; Shahidi, F.; Liyanapathirana, C.M. et al. Turkish Tombul hazelnut (Corylus avellana L.). 1. Compositional characteristics. J. Agric. Food Chem. 51, 3790-3796 (2003).

21) Carosio, S.; Berardinelli, M.G.; Aucello, M. et al. Impact of ageing on muscle cell regeneration. Ageing Res. Rev. 10, 35-42 (2011).

22) Smith, K.; Reynolds, N.; Downie, S. et al. Effects of flooding amino acids on incorporation of labeled amino acids into human muscle protein. Am. J. Physiol. 275, E73-78(1998).

23) Patti, M.E.; Brambilla, E.; Luzi, L. et al. Bidirectional modulation of insulin action by amino acids. J. Clin. Invest. 101, 1519-1529 (1998).

24) Castellino, P.; Luzi, L.; Simonson, D.C. et al. Effect of insulin and plasma amino acid concentrations on leu- cine metabolism in man. Role of substrate availability on estimates of whole body protein synthesis. J. Clin. Invest. 80, 1784-1793 (1987).

25) Chanseaume, E.; Giraudet, C.; Gryson, C. et al. Enhanced muscle mixed and mitochondrial protein synthesis rates after a high-fat or high-sucrose diet. Obesity (Silver Spring) 15, 853-859 (2007).

26) Rincón-Cervera, M.A.; Valenzuela, R.; Hernandez-Rodas, M.C. et al. Supplementation with antioxidant-rich extra virgin olive oil prevents hepatic oxidative stress and reduction of desaturation capacity in mice fed a high-fat diet: Effects on fatty acid composition in liver and extrahepatic tissues. Nutrition 32, 1254-1267 (2016).

27) Accardi, G.; Aiello, A.; Gargano, V. et al. Nutraceutical effects of table green olives: A pilot study with Nocellara del Belice olives. J. Agric. Food Chem. 59, 99359941(2011)

28) Medina-Remon, A.; Tresserra-Rimbau, A.; Pons, A. et al. Effect of total dietary polyphenols on plasma nitric oxide and blood pressure in a high cardiovascular risk cohort. The PREDIMED randomized trial. Nutr. Metab. Cardiovasc. Dis. 25, 60-67 (2015).

29) Rychter, M.; Gaucher, C.; Boudier, A. et al. S-Nitrosothiols-NO donors regulating cardiovascular cell proliferation: Insight into intracellular pathway alterations. Int. J. Bioche. Cell Biol. 78, 156-161 (2016).

30) Del Rio, D.; Calani, L.; Dall'Asta, M. et al. Polyphenolic composition of hazelnut skin. J. Agric. Food Chem. 59, 9935-9941 (2011).

31) Luzi, L.; Castellino, P.; Simonson, D.C. et al. Leucine metabolism in IDDM. Role of insulin and substrate availability. Diabetes 39, 38-48(1990).

32) Surra, J.C.; Barranquero, C.; Torcal, M.P. et al. In comparison with palm oil, dietary nut supplementation delays the progression of atherosclerotic lesions in female apoE-deficient mice. Br. J. Nutr. 109, 202-209 (2013) 